\title{
Column press design optimization on the basis of stress-strain state's mathematical modeling
}

\author{
Sergey Karpushkin ${ }^{1, *}$, Aleksey Glebov ${ }^{1}$, and Sergey Karpov ${ }^{1}$ \\ ${ }^{1}$ Tambov State Technical University, 392000 Tambov, Sovetskaya 106, Russia
}

\begin{abstract}
The problem of optimizing the design of vertical column press traverse according to the metal consumption criterion is taken into consideration. As a limitation, the maximum value of equivalent stresses in the volume of the traverse was used. It is shown that the methods of parametric and topological optimization are ineffective for solving the optimization problem. A mathematical model of the stress-strain state of the traverse is proposed, which allows carrying out simulation independently from other elements of the press. The finite element analysis of the existing structure of the traverse revealed the redundancy of the loaded elements amplification, as well as the low manufacturability of the structure. The method of expert analysis was used to find the optimal design. The effect of the thickness of the base of the traverse on the resulting equivalent stresses was studied. A new design of the traverse was proposed on the base of the study results. That design is characterized by low metal capacity and high manufacturability. It is found that the maximum stresses occur in the areas of rapid changes in shape, as well as in the areas of loads.
\end{abstract}

\section{Introduction}

The column type of presses has become widespread in the industry due to the simplicity of construction, low metal consumption (in comparison with the frame type of presses), as well as ease of operation as molds are available from any of the four sides [1]. Strength calculation of the main elements of the column press (diameter of columns, height of nuts, thread parameters, etc.) is carried out according to known engineering techniques [2]. However, reliable methods for calculating such an element as a upper crossbeam of a column press (see Fig. 1, 2) are not described in the literature due to the complexity of its geometry.

As a rule, the shape and dimensions of the upper crossbeam depend on the "design reasons", which often leads to unreasonable costs of metal for its manufacture. In this connection, the task of optimizing the design of the upper crossbeam of the column press, using the finite element analysis of the stress-strain state (SSS) is relevant. A common feature of the known works in this area is the use of the finite element method as a tool for verifying calculations of the already existing press designs $[3,4]$. The tasks of developing and optimizing new designs are not given much attention.

* Corresponding author: karp@mail.gaps.tstu.ru 


\section{Mathematical model of stress-strain state of column press upper crossbeam}

The vector field $\mathbf{u}$ of body displacements under the influence of deformations caused by external forces is described by the differential equation of Lame equilibrium [5]:

$$
\frac{2(1-v)}{1-2 v} \operatorname{grad}(\operatorname{div} \mathbf{u})-\operatorname{rot}(\operatorname{rot} \mathbf{u})=0
$$

where $v$ is Poisson's ratio.

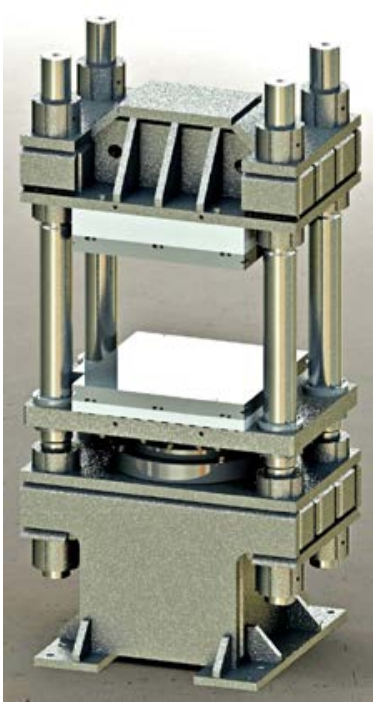

Fig. 1. General view of column press.

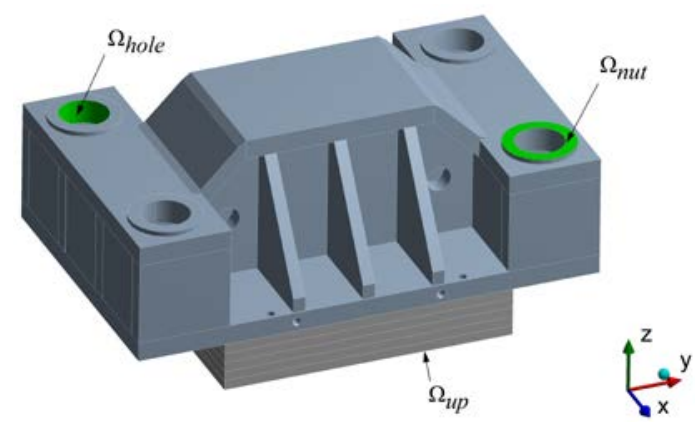

Fig. 2. Upper crossbeam of the column press.

Under the influence of the load, the upper crossbeam may slightly move along the columns that serve as guides. We set the condition for the absence of displacements along the $x$ and $y$ axes on cylindrical surfaces $\Omega_{\text {hole }}$ (see Fig. 2) of upper crossbeam hole:

$$
u_{x}=\left.0\right|_{\Omega_{h o l e}} ; u_{y}=\left.0\right|_{\Omega_{\text {hole }}} .
$$

Vertical movement of the upper crossbeam is limited by the column nuts. Consequently, the boundary condition in the region $\Omega_{n u t}$ of the upper crossbeam contact with the nuts will have the form

$$
u_{z}=\left.0\right|_{\Omega_{n u t}}
$$

External load is transferred to the upper crossbeam from the hydraulic cylinder through a package of plates and molds. We assume that there is no friction between the upper heating plate, the heat insulating plates, the column nuts and the base of the beam. a monolithic system is considered. Without taking into account the geometry of the specific molds, their number and location on the lower plate, it can be assumed that the load is distributed evenly over the working surface $\Omega_{u p}$ of the upper plate of the press, rigidly connected with the upper crossbeam through the layer of heat-insulating plates: 


$$
\sigma_{z}=\left.\frac{P}{F}\right|_{\Omega_{u p}},
$$

where $\sigma_{z}$ is $z$-component of normal stresses, $\mathrm{Pa}$;

$P$ is the force developed by the hydraulic cylinder, $\mathrm{N}$;

$F$ is the working surface area of the press plate, $\mathrm{m}^{2}$.

To simulate the effect of molds on the design syste $\mathrm{m}$, we add the condition of the surface non-deformability $\Omega_{u p}$ in the vertical direction (this condition is relevant to the case when all plate working surfaces are filled with with molds):

$$
u_{z}=\text { const }\left.\right|_{\Omega_{u p}} .
$$

Vectors of normal stresses $\boldsymbol{\sigma}$, tangential stresses $\boldsymbol{\tau}$ and displacements $\mathbf{u}$ are connected through linear deformations $\boldsymbol{\varepsilon}$ and angular deformations $\gamma$ :

$$
\begin{gathered}
\varepsilon_{x}=\frac{\partial u_{x}}{\partial x} ; \varepsilon_{y}=\frac{\partial u_{y}}{\partial y} ; \varepsilon_{z}=\frac{\partial u_{z}}{\partial z} ; \\
\sigma_{x}=\frac{E v\left(\varepsilon_{x}+\varepsilon_{y}+\varepsilon_{z}\right)}{(1+v)(1-2 v)}+\frac{E \varepsilon_{x}}{1+v} ; \\
\sigma_{y}=\frac{E v\left(\varepsilon_{x}+\varepsilon_{y}+\varepsilon_{z}\right)}{(1+v)(1-2 v)}+\frac{E \varepsilon_{y}}{1+v} ; \\
\sigma_{z}=\frac{E v\left(\varepsilon_{x}+\varepsilon_{y}+\varepsilon_{z}\right)}{(1+v)(1-2 v)}+\frac{E \varepsilon_{z}}{1+v} ; \\
\gamma_{x}=\frac{\partial u_{y}}{\partial x}+\frac{\partial u_{x}}{\partial y} ; \gamma_{y}=\frac{\partial u_{z}}{\partial y}+\frac{\partial u_{y}}{\partial z} ; \gamma_{z}=\frac{\partial u_{x}}{\partial z}+\frac{\partial u_{z}}{\partial x} \\
\tau_{x}=\frac{E \gamma_{x}}{2(1+v)} ; \tau_{y}=\frac{E \gamma_{y}}{2(1+v)} ; \tau_{z}=\frac{E \gamma_{z}}{2(1+v)},
\end{gathered}
$$

where $E$ is Young's modulus of elasticity, $\mathrm{Pa}$.

As a criterion for evaluating the strength of structures, the concept of equivalent stresses in the fourth strength hypothesis is most often used. The calculated values of the equivalent stresses should not exceed the yield strength of the material used $\left[\sigma_{T}\right]$ when taking into account the strength margin (factor)

$$
K \varepsilon_{e q v}<\left[\sigma_{T}\right]
$$

where $K$ is strength factor;

$$
\varepsilon_{e q v}=\sqrt{\frac{1}{2}\left[\left(\sigma_{1}-\sigma_{2}\right)^{2}+\left(\sigma_{2}-\sigma_{3}\right)^{2}+\left(\sigma_{3}-\sigma_{1}\right)^{2}\right]}
$$

is equivalent stress, $\mathrm{Pa}$;

$\sigma_{1}, \sigma_{2}, \sigma_{3}$ are main stresses, Pa.

The main stresses are the roots of the characteristic equation of the stressed state [6] 


$$
\sigma_{v}^{3}-\sigma_{v}^{2} J_{1}-\sigma_{v} J_{2}-J_{3}=0
$$

where $J_{1}=\sigma_{x}+\sigma_{y}+\sigma_{z}$;

$$
\begin{gathered}
J_{2}=-\sigma_{x} \sigma_{y}-\sigma_{y} \sigma_{z}-\sigma_{z} \sigma_{x}+\tau_{x}^{2}+\tau_{y}^{2}+\tau_{z}^{2} \\
J_{3}=\sigma_{x} \sigma_{y} \sigma_{z}+2 \tau_{x} \tau_{y} \tau_{z}-\sigma_{x} \tau_{y}^{2}-\sigma_{y} \tau_{z}^{2}-\sigma_{z} \tau_{x}^{2} .
\end{gathered}
$$

It is assumed that $\sigma_{1}>\sigma_{2}>\sigma_{3}$.

Thus, the problem of optimizing the design of the upper crossbeam of the column press can be formulated as follows: it is necessary to find such structural characteristics of the upper crossbeam (thickness of the main plate, reinforcement scheme, thickness and the method of coupling of all reinforcement elements) that ensure the minimum value of its mass if the conditions of the mathematical model of the stress-strain state (1)-(12) are satisfied.

\section{Upper crossbeam optimization}

In cases, if all variable variables are defined, the areas of their definition are known and an adequate mathematical model of the object of investigation is constructed, we can apply any of the traditional methods of optimization.

Since the analysis of the objects described by partial differential equations requires considerable CPU time, it is quite effective to use the theory of experimental design [7]. According to this theory, a complex objective function is replaced by a multidimensional (in terms of the number of variable variables or factors) polynomial. In most cases, it is sufficient to construct a polynomial of the second degree. Calculation of the coefficients of the polynomial is carried out by the regression analysis, in particular, using the method of least squares. For this, a series of computational experiments is carried out at strictly defined points of the factor space, the totality of which is called the experimental design. It was shown in [7] that when optimizing the temperature fields of the heating plates of presses, a high efficiency (accuracy of approximation) was demonstrated by the BoxBehnken non-compositional design and the rotatable design, whose informational function is an isosurfaces in the form of concentric hyperspheres.

The solution of this optimization problem is complicated by the fact that variable variables are not explicitly known, and their formalization is quite challenging. For example, it is extremely difficult to describe such a parameter as a "reinforcement scheme" using a set of scalar variables.

Topological optimization is currently a popular method for solving such strength problems [8]. The idea of this method is based on the search for the best distribution of material in a given volume: lightly loaded areas of the structure that do not contain technological elements (for example, fixing holes) are gradually reduced, thereby reducing the total mass. The method of topological optimization is implemented in some modern systems of engineering analysis. The result of its application is the image of the design, containing elements with irregularly shaped surfaces. For this reason, subsequent design and processing of the results is required. The method of topological optimization is highly efficient when using additive technologies for which there are no strict process limitations (it is possible to manufacture parts containing cavities of arbitrary shape) [9]. With regard to presses, topological optimization is not always justified, and the solutions obtained are often non-technological [8].

In this paper, the method of expert analysis was used to solve the optimization problem. At the first stage, the structure of the existing upper crossbeam was analyzed, its shortcomings were revealed. The second stage was the development of a new design meeting the requirements of strength, low metal content and manufacturability. A step-by- 
step method of development with intermediate analysis of results was applied: the structural elements were added gradually, with strengthening of the most loaded areas.

\subsection{Analysis of the existing design}

In Fig. 2 shows the upper crossbeam of a column press for the production of rubber products manufactured by corporation "Zavod Tambovpolimermash", Tambov, Russia. This design was analyzed in accordance with the presented mathematical model. To solve equations (1) (12), we used the ANSYS finite element analysis system. he geometry of the upper crossbeam was approximated by SOLID186 20-node finite elements of the second order (see Fig. 3) with three degrees of freedom: $u_{x}, u_{y}, u_{z}[10]$.
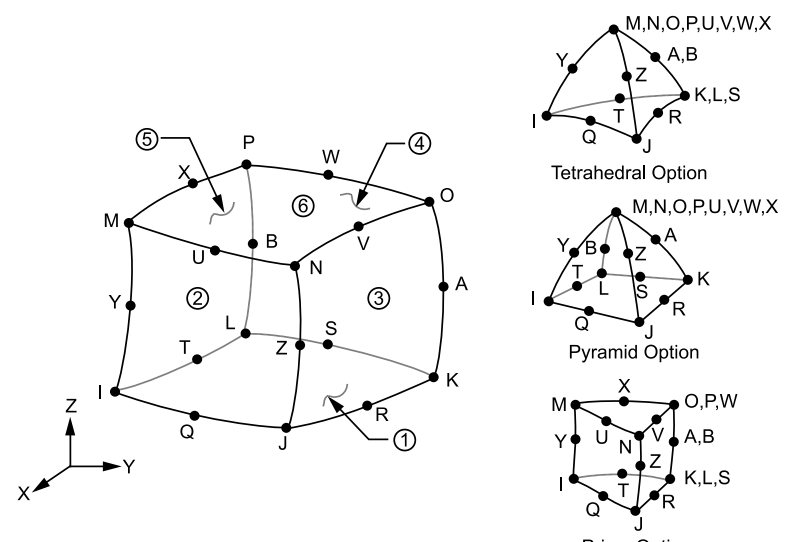

Prism Option

Fig. 3. ANSYS SOLID186 finite element.

The upper crossbeam is made of steel $40, E=2 \cdot 10^{11} \mathrm{~Pa}, v=0,28$. Nominal press force $P=2,5 \cdot 10^{6} \mathrm{~N}$.

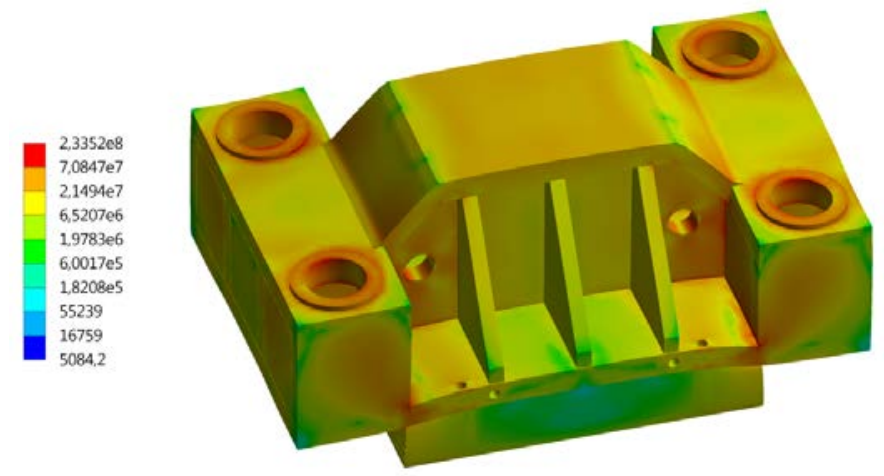

Fig. 4. The equivalent stress field of the existing upper crossbeam.

Fig. 4 shows the equivalent stress field of the upper crossbeam on a logarithmic scale. The maximum stresses were $71 \mathrm{MPa}$. We note that the areas of stress concentrators were not taken into account, since the calculated values in these areas can significantly exceed the true stresses due to accepted assumptions (no friction between the press elements). The yield point of steel 40 is $330 \mathrm{MPa}$. Thus, with a mass of $538 \mathrm{~kg}$, the strength factor is 4.65 . The current design of the upper crossbeam meets the requirements of strength, but it has a 
high metal capacity. Low manufacturability due to the large total length of the welded seam is also a disadvantage of this design.

\subsection{Development of a new design}

The simplest design of the upper crossbeam, which is a flat plate with the dimensions $600 \times 600 \times 20 \mathrm{~mm}$ (see Fig. 5) was used for the development of a new design.

The results of calculating the equivalent stresses for varying the plate thickness in the range from 20 to $60 \mathrm{~mm}$ are summarized in Table. 1. A graphical representation of the results is shown in Fig. 6.

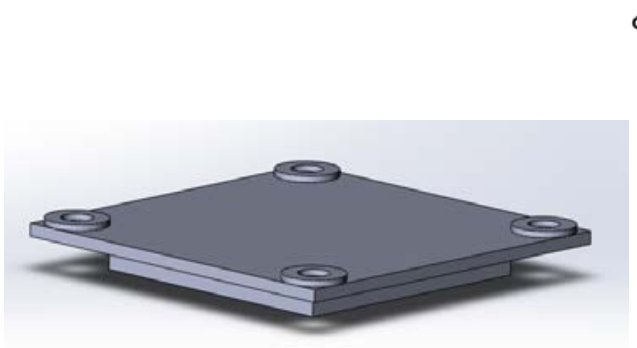

Fig. 5. Initial design of upper crossbeam.

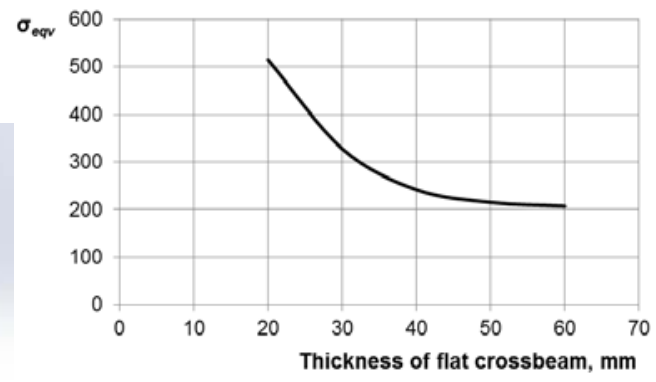

Fig. 6. Dependence of maximum equivalent stresses on the thickness of flat upper crossbeam.

Table 1. Optimization of flat upper crossbeam.

\begin{tabular}{|c|c|c|c|c|}
\hline Thickness, $\mathrm{mm}$ & Mass, $\mathrm{kg}$ & Maximum stress, MPa & Strength factor & Max displacement, $\mathrm{mm}$ \\
\hline 20 & 129 & 515 & 0.64 & 0.42 \\
\hline 30 & 156 & 328 & 1.01 & 0.29 \\
\hline 40 & 184 & 242 & 1.36 & 0.22 \\
\hline 50 & 211 & 215 & 1.53 & 0.18 \\
\hline 60 & 239 & 207 & 1.59 & 0.15 \\
\hline
\end{tabular}

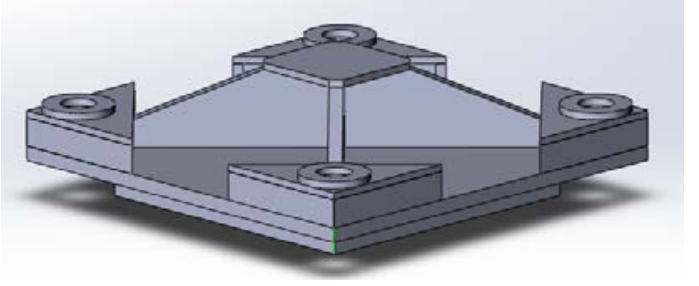

Fig. 7. Final design of upper crossbeam.

From the results obtained it follows that the strength condition is satisfied for flat upper crossbeam s with a thickness of more than $30 \mathrm{~mm}$. With an increase in thickness over 40 $\mathrm{mm}$, the growth rate of the strength factor is significantly reduced, but the mass increases linearly. Consequently, the use of upper crossbeam with a base thickness of more than 40 $\mathrm{mm}$ is irrational.

A gradual increase in the rigidity of the upper crossbeam by adding reinforcing elements made it possible to propose the design shown in Fig. 7. For this upper crossbeam, the maximum equivalent stresses were $181 \mathrm{MPa}$ at a mass of $170 \mathrm{~kg}$. Thus, with approximately the same mass for this design, the strength factor was 33\% higher than for a flat upper crossbeam with a thickness of $40 \mathrm{~mm}$. The existing upper crossbeam exceeds the mass of a new design more than 3 times. However, the proposed upper crossbeam does not 
have such a high strength factor that it can become critical when testing presses with pressures exceeding the nominal pressure.

\section{Results and discussion}

A mathematical model of the stress-strain state of the upper crossbeam of the column press has been developed, which makes it possible to conduct strength analysis independently of other press elements.

Based on the calculation of the stress-strain state, the shortcomings of the existing upper crossbeam design have been revealed.

The problem of optimizing the column press upper crossbeam according to the criterion of metal content has been formulated. The solution of the problem has been found using the method of expert analysis. The method of topological optimization looks promising for the search for the best design, but it is difficult to implement due to the requirement of manufacturability.

The analysis of the calculation results of equivalent stresses has shown the need to take into account the nonlinear effects of the interaction of parts of the design (the presence of friction) in further studies.

This work was financially supported by Ministry of Education and Science of the Russian Federation within the basic part (project 8.7082.2017/8.9)

\section{References}

1. E.N. Malygin, M.N. Krasnyanskiy, V.G. Mokrozub. MATEC Web of Conferences. 129, 01008 (2017).

2. V.K. Zavgorodnij, E.L. Kalinchev, E.G. Maharinskij. Oborudovanie predpriyatij po pererabotke plastmass [Equipment for plastics processing plants] (L.: Himiya, 1972)

3. V.I. Ganush, N.P. Ostroverhov, A.V. Sultan i dr. Metallurgicheskaya i gornorudnaya promyshlennost' [Metallurgical and mining industries], 7, 121-127 (2015)

4. Y. Li, T. Wang, ICMA, 1301-1306 (2015)

5. A.T. Bikmeev, R.K. Gazizov, V.YU. Ivanov i dr. Vestnik YUUrGU [South Urals State University Bulletin], 25, 64-75 (2011)

6. I.A. Birger, R.R. Mavlyutov. Soprotivlenie materialov [Strength of materials] (M.: Nauka, 1986)

7. A.V. Ostroukh, A.O. Glebov, S.V. Karpov et al. American Journal of Applied Sciences, 11, 6, 939-946 (2014)

8. Z.D. Duan, J.J. Wu. Applied Mechanics and Materials, 44, 1828-1832 (2011)

9. D. Brackett, I. Ashcroft, R. Hague R. Proceedings of the solid freeform fabrication symposium, 1, 348-362 (2011)

10. A.M. Wagih, M.M. Hegaze, M.A. Kamel. AIAA SPACE and Astronautics Forum and Exposition (2017) 